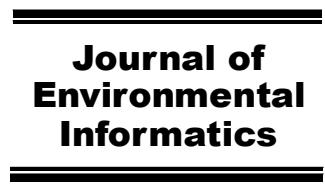

www.iseis.org/jei

\title{
A Web-Based Collaborative System for Remote Monitoring and Analysis of Livestock Farm Odours
}

\author{
R. Liu, L. L. Pan, and S. X. Yang ${ }^{*}$ \\ School of Engineering, University of Guelph, Guelph, Ontario N1G 2W1, Canada
}

Received 15 October 2008; revised 12 March 2009; accepted 1 April 2009; published online 10 June 2009

\begin{abstract}
Monitoring and analysis of livestock farm environments require collection and management of large amount of data from distributed farms. There is an increasing demand for collaboration among livestock producers, environment agencies and governments. This paper presents a collaborative system for monitoring and analyzing livestock farm odours remotely, and for enhancing the collaboration among users. This system utilizes a web-based portal application as the infrastructure for running distributed applications. Livestock farm odour information is stored in central servers. Distributed users can access the data remotely, submit odour data for analysis, receive analysis results through the Internet, exchange information, and discuss odour related topics on public forums. This collaborative system provides a collaborative, robust, and user-friendly environment for distributed users to efficiently manage and process the data records, share the analysis results and other information.
\end{abstract}

Keywords: collaborative web-based application, livestock farm odour, remote monitoring and analysis

\section{Introduction}

Odour emissions from livestock facilities have caused serious complaints from rural residents. The Ontario governments have worked on setting up regulations and policies to limit odorous air emissions from livestock farms. Environment agencies and researchers have made significant efforts to investigate and resolve the livestock farm odour problems (Janes et al., 2004; Pan et al., 2007; Pan and Yang, 2007a, 2009).

In order to efficiently resolve the livestock farm odour problem, there is an increasing demand for collaboration among livestock producers, consulting agencies and governments (federal, provincial and local). Through collaboration, they can share and make better use of their professional skills and expertise to serve the odour control problem. However, these users have different backgrounds, are responsible for different tasks based on their roles, and work at different locations. It is difficult to bring them together to achieve prompt and convenient information exchange and communications. High speed Internet provides a way for remote communications and data sharing. However, traditional HTML-based content only provides single public-facing web site. It is enough for small organizations to advertise their services or products, but it cannot satisfy the collaboration requirements of such a large-scaled service, management, industrial and research community.

\footnotetext{
* Corresponding author. Tel.: +1 519 8244120; fax: +1 5198360227.

E-mail address: syang@uoguelph.ca (S. X. Yang).
}

ISSN: 1726-2135 print/1684-8799 online

(C) 2009 ISEIS All rights reserved. doi:10.3808/jei.200900142
By using the traditional HTML-based web technologies, it requires hundreds or even thousands web sites for all the users and activities in this odour monitoring and analysis task. It is expensive and time consuming to keep up with the need to create and manage those web sites.

Due to the rapid development of platform-independent and central processing technologies, web-based applications are emerging to provide collaboration among distributed users. In such a web-based application, the essential software and database are located on a central server and are accessible over networks. Therefore, users can upload and process their odour data through Internet web browser. Many web-based applications have been developed in various areas, such as education (Chang et al., 2003; Jackson, 2003; Wang et al., 2004), information technology (Yu et al., 2003; Taguchi and Tokuda, 2005), urban planning (Counsell, 2004; Babar and Gorton, 2004), and environment monitoring (Zeng et al., 2007; Athanasiadia and Mitkas, 2007; Carswell et al., 2008). Yu et al. (2003) developed a web-based application for 3D visualization and collaboration to enable production, dissemination and use of 3D imagery and geospatial information on a hierarchical level. Counsell (2004) developed a web-based application for collaboration on managing and maintaining large area urban modeling. Taguchi and Tokuda (2005) designed a web application generator to generate data-intensive web applications by using diagrams that represent data-flow relationships among web components. Maglogiannis et al. (2006) presented a collaborative web-based application for medical diagnosis over the Internet via peer-to-peer distribution of electronic health records. Yu and Cheng (2007) developed a web-based decision support system for slopeland hazard warning, which combines with GIS 
technology and can provide real-time monitoring of rainfall via Internet Explorer. Despite broad applications of web collaboration technologies, none of them were developed for collaboration and cooperation among livestock producers and environment organizations. Zeng et al. (2007) proposed a web-based system for managing the information of urban water resource social renewability. An artificial neural network was developed for analysis of the information and the evaluation results were published via the Internet. Carswell et al. (2008) introduced a web-based mobile system for monitoring fish species at risk. The fish data is stored in a central database and is delivered to GPS-enabled hand-held devices so that professionals can access the data remotely and make decision immediately.

To date, however, the information exchange among livestock farmers, environment agencies and governments on resolving odour issues is still quite limited. The objective of this study is to develop a web-based collaborative framework to assist the collaborations in resolving the livestock farm odour problems. Collaboration, in dictionary, is defined as a process that multiple people/organizations work together to achieve an intersection of common goals (Oxford, 1989). Collaborative software in information technology is an application designed for people participated in a common task to reach their goals. The proposed approach provides communication and data-sharing capability among distributed users, and thus enhances collaboration and cooperation among users with different backgrounds for more efficient odour management and decisionmaking. All the data collection, processing and analysis tools are developed in a web-based application, and can be accessed simultaneously by multiple users. Users can communicate with each other and share information from distributed locations. This proposed collaborative framework is built on Microsoft Framework 2.0 and Windows SharePoint system. Visual Studio 2005 is chosen as the development environment, and Microsoft SQL server 2005 is used as the data repository.

\section{The Proposed Approach}

The proposed collaborative framework should provide essential collaboration capabilities for effective information sharing, data processing and management. This framework is developed for collecting, monitoring, processing, and analyzing livestock farm odour data. This solution not only provides robust data management and data integration for the odour research community, but also brings added values to the experimental data. However, the development is complex. The following aspects are considered:

(1) Multiple user collaboration: In general, the collaborative framework should be able to serve multiple purposes, such as user collaboration, data processing, and information sharing. The odour data that generated by a client acquisition system and data analysis results are shared by governments and authorized agencies. Farmers can select environment agencies to analyze their odour data, and grant necessary permissions to chosen agencies to access their data. Farmers also have access to the analysis results and feedbacks from agencies. Ag- encies process and analyze the data, generate result reports, provide suggestions on odour control practice. Governments review the odour data and analysis results, provide guidance for farm operations, and enforce environmental regulations. Governments also consult with agencies to revise or improve environmental laws or regulations. Users can interact with each other either by correspondence via private emails, or by discussions threads on the public discussion board. The proposed collaborative framework provides a disciplined approach to facilitate the collaboration among livestock farmers, agencies and governments.

(2) Central data management and security requirement: Generally, odour data are collected from separate farms and stored in distributed computers. In order to analyze those distributed data and generate results, the data records need to be sent into a central data server, where the data analysis system can process numerous users' requests simultaneously. However, in this way security issue is arisen, due to the sensitive and private nature of the odour information. In order to solve the security problem, separate data record libraries are established on the central data server, and are assigned to different users. Security settings and policies are specified for sensitive information. The collaborative framework authorizes different access privilege to different users, based on their roles and responsibilities. Only authorized users have access to the content in the record library via role-based permission control; unauthorized users have no access to private contents unless the owner of the record library grants them appropriate access privilege. For example, livestock farmers do not have access to the data of other farmers unless they are granted access. This feature enhances the data security.

(3) User-friendly interface: The user interface of the system provides a user-friendly and consistent environment for users with different backgrounds and expertise. User interface in computer science is a place for human-computer interaction. It provides input and output means for users to access the computer system. Users can control the system by keystrokes with the computer keyboard, or by movements and clicking of the computer mouse; the system presents the users with graphical, textural and auditory information. Considering most potential users of the application are not computer experts, it is very important that the system should provide a user-friendly interface, adopting all necessary functionality that the users need in operations and collaborations. This collaborative framework is a platform-independent web-based application, which can be run on different platforms, such as Windows, UNIX, and Linux. Users do not need to have the same platform on their computers. All users can access the real-time interactive collaborative system through a web browser. Moreover, all data acquisition applications are installed on the central server and have online connection to the central server, so that individual users do not have to process the data on their own computers. These considerations make the proposed web-based application very easy to use, and also provide great convenience for the system administrators to maintain and update the system, since they only need to modify or tune applications on the central server. In addition to the access by desktop personal com- 
puters, the interface also provides support for mobile device access.

(4) Transparent workflow: The collaborative framework is a workflow-driven application, supporting users to submit different tasks for processing data records. Workflows represent the operational information of a work procedure, such as how processes are structured, formalized, enforced, audited, and how they are synchronized. With the robust, customizable workflow framework, users can tell whether a process request has been created, reviewed, processed, or updated, and also receive the analyzed information. The central workflow-enabled processing functionality helps users to make effective decisions by providing all useful information in one central place.

\section{Design of the Web Based Collaborative Framework}

The collaborative system is built on top of the wireless sensor networks that are deployed on livestock farms to collect odour data. The architecture of the collaborative system is shown in Figure 1. The system consists of three main components: client applications for wireless sensor networks; a central database server; and central application servers.

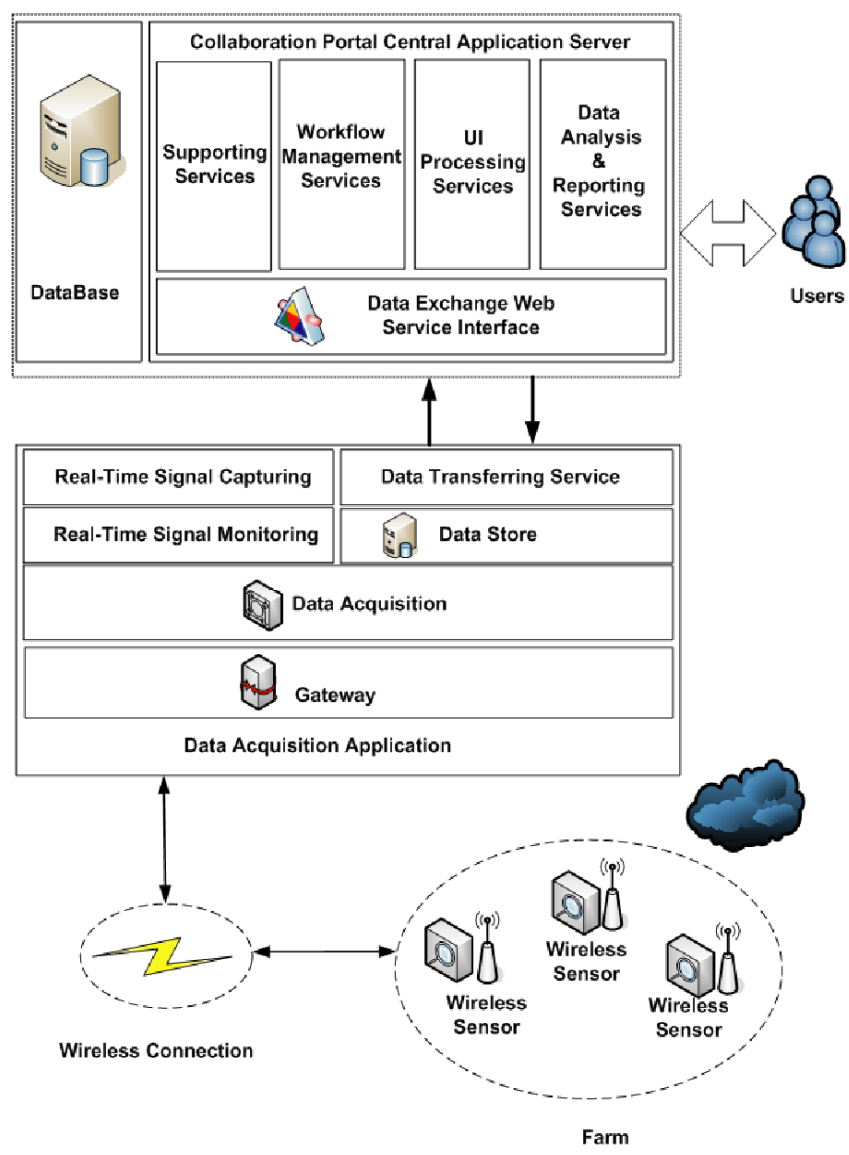

Figure 1. Architecture of the collaborative web-based application.

\subsection{Client Applications for Wireless Sensor Networks}

The wireless sensor networks are at the lowest level of the collaborative system. They are deployed on livestock farms to collect odour data. Each wireless sensor network has many sensor nodes, where each node is an electronic nose (e-nose) that is built of gas sensors and environmental sensors to measure odorous compounds and temperature and relative humidity. Sensor signals are delivered to and stored on a local computer for future processing. The detailed information about the wireless sensor networks can be found in Pan and Yang (2009), and the development of electronic noses is described in details in Pan and Yang (2007a). The wireless sensor networks provide the collaborative system with remote odour monitoring ability.

Client applications are developed to assist the data collection for the wireless sensor network. A client application includes three main components: a data acquisition module to acquire the sensor signals transmitted to the computer via the wireless network; a client-side database that stores the data, and a data transfer web service that exchanges information between the client-side computer and the central database server.

\subsubsection{Data Acquisition Module}

The sensor signals collected on a farm are transmitted through the wireless network to a gateway, which receives and collects the digital signals. Then the data acquisition system in the computer interfaces with the getaway and gathers the digital signals into the client-side database.

\subsubsection{Client-Side Database}

Database plays an important role as data storage, management, and exchange center. In this system, data structures (records and files) are optimized to deal with very large amounts of data stored on a data storage server. A database based on Microsoft SQL 2005 database Server 2005, is developed for storing sensor signals and odour information. Besides database itself, there is also a data management system to store and organize or pre-process the data. Database management system is a complex set of software modules that help the organization, storage and retrieval of data in the database. It can connect to the database, load data acquisition console, and display sensor signals dynamically.

The interface of data display system is shown in Figure 2. It provides both on-line and off-line signal display options. Several display settings can be selected by users, such as different update interval, number of visible points and data range, etc. This would be very helpful for routine operation and various studies.

\subsubsection{Data Transfer Web Service}

The client application uses a web service to interface with central servers. The web service feeds data to or consumes data from central servers. It provides remote accessibility for the client application to integrate with the central servers. 


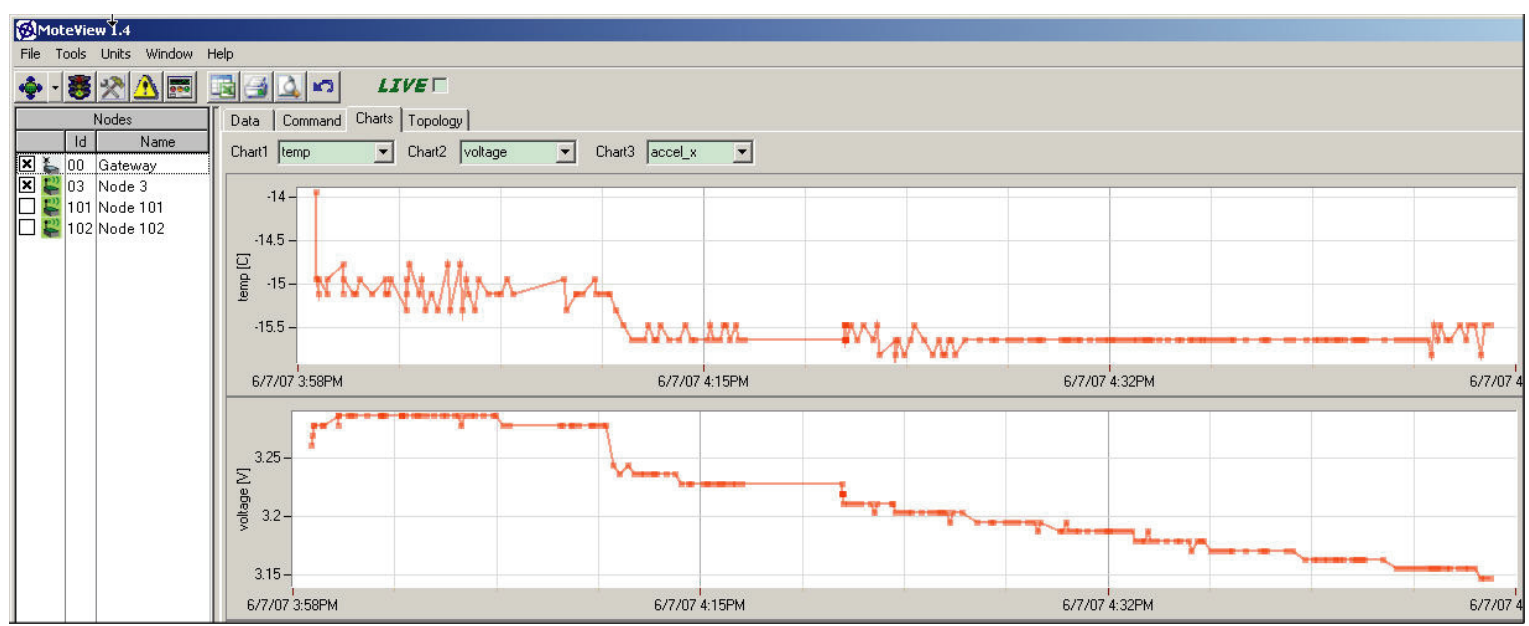

Figure 2. The dynamic monitoring console.

\subsection{Central Database Server}

The odour data collected by the wireless sensor networks from farm terminals are shared among decision makers, such as governments and environment agencies. The central database server provides data/information storage for the collaborative system. The proposed web-based application uses the central SQL Server 2005 as central data repository. SQL Server 2005 provides enhanced safety and security, high performance and scalability, as well as a wide range of database service tools. The central database stores the data, and provides the data to data processing services. Figure 3 shows the data structure of the central database. The central database stores different kinds of data sets and information such as real-time odour data that are collected online by the gas sensors on livestock farms, historical data records, farm information and user profiles, analysis results, and search index. With all these integrated database capabilities, the collaborative system can also help the researchers to improve data analysis and research efficiency.

\subsection{Central Application Servers}

The architecture of the central application server is shown in Figure 4. It includes three application servers: a collaboration server, an application server, and an active directory server. The collaboration server provides a platform for data and information integration, sharing and exchange, as well as collaboration and cooperation ability among distributed end users. The application server is used to host data processing and analysis services to provide core data processing, reporting and content crawling functionality. All data processing features are shared across application domains through web services. The active directory server stores user information, and provides user authentication and authorization functionality.

Livestock farmers, environment agencies, and government users are the designed users of the application server. The application server consists of two basic components: a data processing tier, and a user collaboration interface. The collaboration interface is the front-end of the application server that

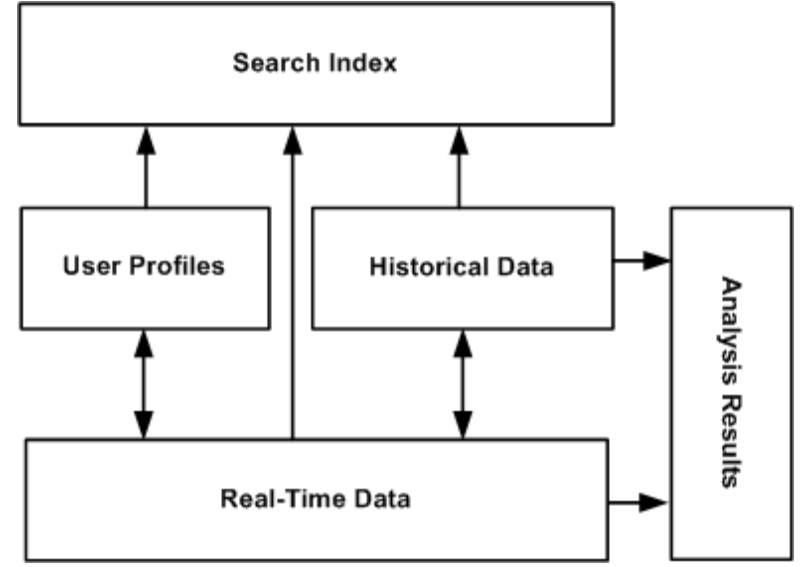

Figure 3. Data structure of the central database server.

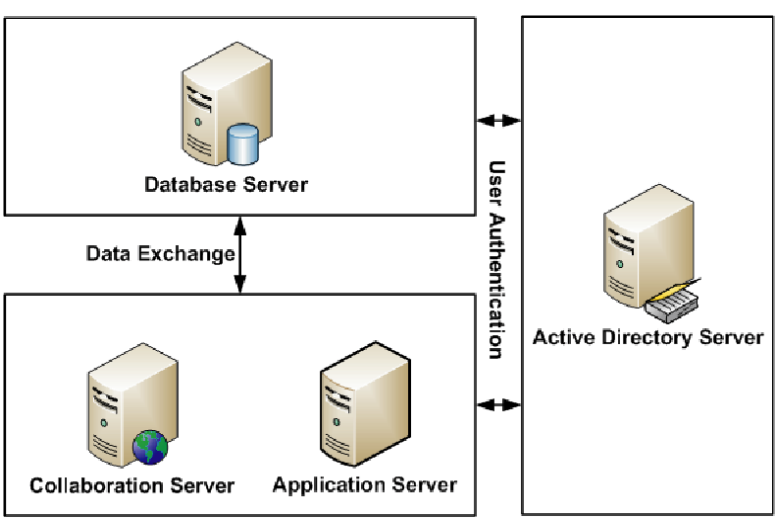

Figure 4. Architecture of the central application server.

contains all the user interfaces and directly interacts with users. Data processing service communicates with the central database server, and the user collaboration interface consumes the data processing results. All the data processing and analysis modules are located in the data processing module. Data processing service receives requests from users through the in- 


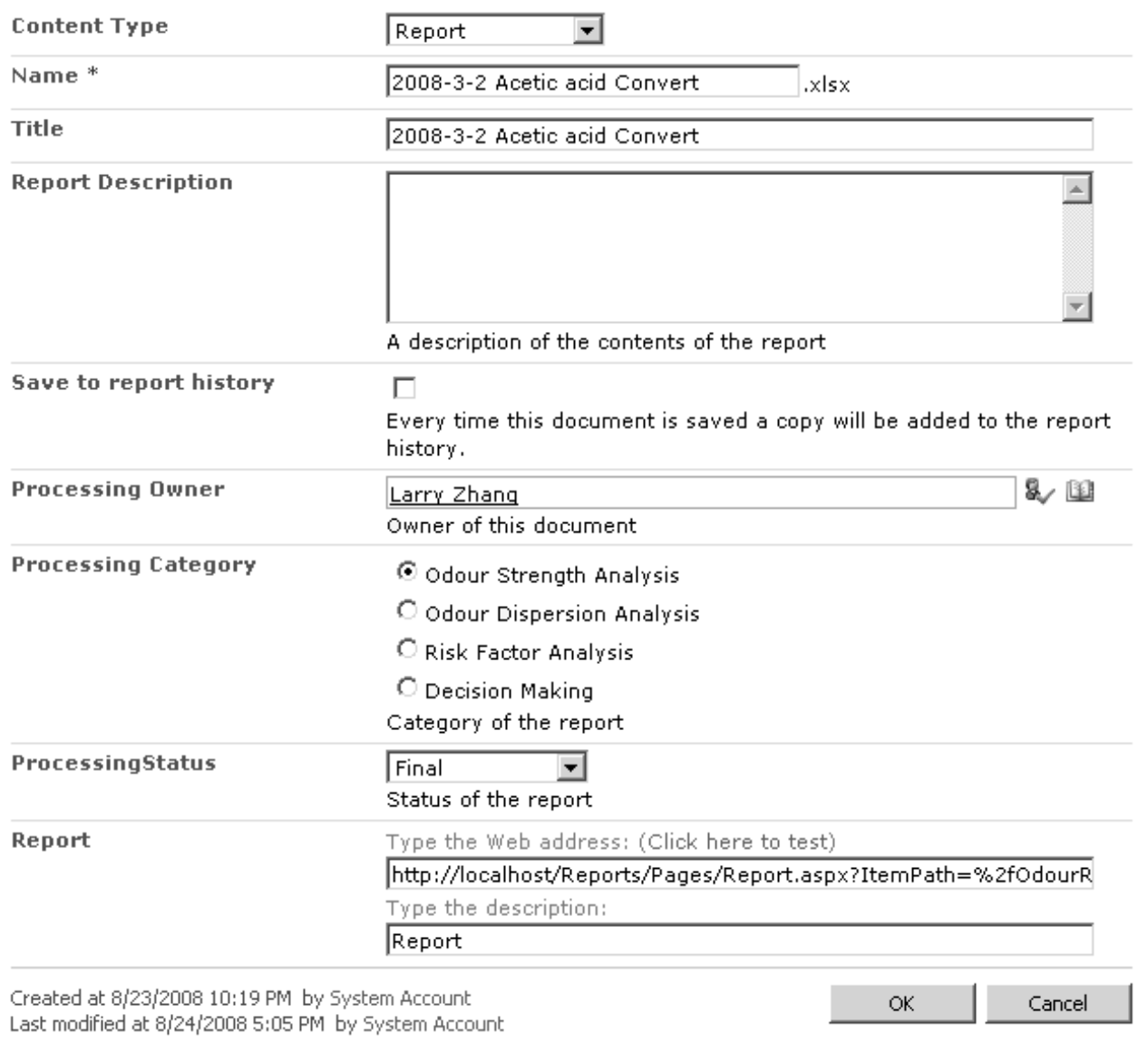

Figure 5. Interface of the data processing services.

terface, looks up information in the database, analyzes the data, processes the requests, generates analysis result reports, and then sends back result reports to presentation layer where the result reports are displayed.

\section{(1) Data Analysis and Reporting Module}

The data analysis and reporting module provides a userfriendly environment and supports data processing operations. This module combines data analysis services and result reporting services. Figure 5 illustrates the framework of the data analysis service that is built as an inter-operable middle-tier of the collaborative system. The collaborative system utilizes the data analysis services to identify rules and patterns in the data, to inform the users where has odour and how strong the odour is, to give suggestion on odour control practice, and to predict the resulted odour strength if any odour control means are applied. The data analysis module has many sub-modules: odour strength analysis module calculates odour concentration/intensity using artificial intelligent techniques; odour dispersion analysis module calculates odour dispersion plume using odour dispersion models based on odour emission rates, topological and meteorological information; risk factor analysis module identifies significant risk factors that cause odour problems; and decision making module assists users to make odour control decisions based on analysis results and human expert know- ledge. The detailed information about the odour dispersion analysis modules, risk factor analysis module, and decision making module can be found in Pan and Yang (2009). The report generation module generates the analysis result reports and provides report accessibility and management.

Neural network methods are used in the data analysis module to explore the odour data pattern. Neural network algorithms mainly address the classification task. They can find nonlinear relationships among the input values of the predictable attribute, by creating an internal classification and regression models that are iteratively improved, based on the actual value (Haykin, 1999). Existing research demonstrates that neural networks can be successfully applied to odor data analysis (Janes et al., 2004, 2005; Pan et al., 2006; Pan and Yang, 2007b).

The odour model used in the data analysis module has three layers as shown in Figure 6: the input layer, the hidden layer and the output layer. The input nodes form the first layer of the neural network, where each input node is mapped to one sensor data input channel such as temperature, humidity or odour sensor data. The second layer is the hidden layer that has hidden nodes, which receives input data from the input layer. The output layer presents the predictable attributes. In this case, our neural network has one single output attribute that is the odour strength. 
The neural network is a feed-forward network. Before using the neural network to provide prediction on odour strength value, it needs known data to tune the network, which is called the training process that is to find the best set of weights for the neural network. The weights of the network are randomly initialized at the beginning. In every training iteration, the network compares the actual value of the training case with the predicted value, and calculates the error at the outputs. Then the weights of the network are adjusted using the error back-ward propagation learning technique. The reason to choose neural network algorithms as the data analysis algorithm is that neural networks can handle complex as well as large amounts of training data efficiently.

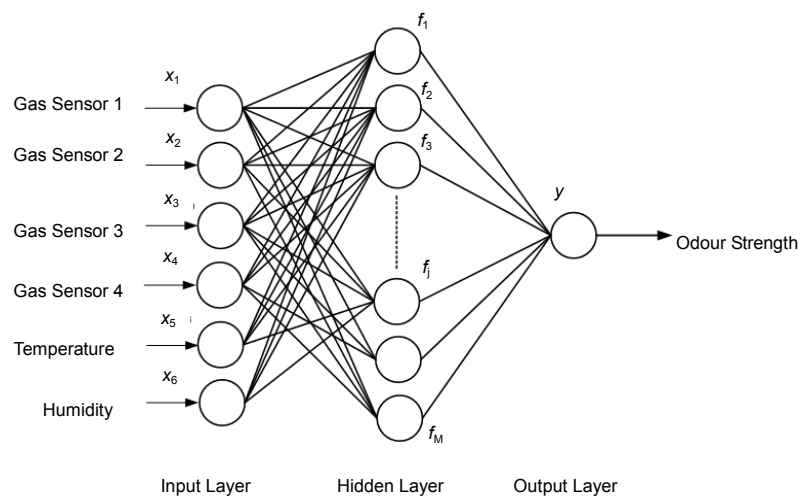

Figure 6. The structure of the neural network.

The processing unit in the neural network is a single neuron node. Each node processes the combination of weighted sum of the input values into a single output value. The logistic function $g(a)$ is selected as the activation function for the hidden nodes, and the tahn function $\varphi(a)$ is selected as the activation function for the output node, which are defined as:

$g(a)=\frac{e^{a}-e^{-a}}{e^{a}+e^{-a}}$

$\varphi(a)=\frac{1}{1+e^{-a}}$

Therefore, the outputs of hidden neurons and the output neuron are given as:

$f_{j}=g\left(\sum_{i=0}^{N} w_{i j} x_{i}\right) j=1,2, \ldots, M$

$y=\varphi\left(\sum_{j=0}^{M} v_{j} f_{j}\right)$

where $y$ is the output of the neural network; $f_{j}$ is the output of the $j$-th hidden node; $w_{i j}$ is the weight between $i$-th input node and the $j$-th hidden node; $v_{j}$ is the weight between the the $j$-th hidden node and the output neuron, $N$ is the number of input nodes, and $M$ is the number of hidden neurons. This neural network updates the weights after a sample case is analyzed. In order to minimize the training error, the least mean square error $E$ is used as the error function:

$E=e^{2} / 2=(t-y)^{2} / 2$

where $t$ is the actual (desired) value of the output neuron for the processed the training sample, and $e$ is the error at the output neuron.

The weight adjusting equations are given as:

$$
\begin{aligned}
& w_{i j}^{\text {new }}=w_{i j}^{\text {old }}+\eta f_{j}\left(1-f_{j}\right) x_{i} e_{j}^{h}, \\
& v_{j}^{\text {new }}=v_{j}^{\text {old }}+\eta\left(1-y^{2}\right) f_{j} e
\end{aligned}
$$

where $\eta$ is the learning rate, and $e_{j}^{h}$ is the error of $j$-th hidden neuron given by:

$e_{j}^{h}=v_{j} e$

The parameters of the neural network such as learning rate $\eta$ and number of hidden nodes are determined by trial and error. The value of $\eta$ is usually set to a large value at the beginning stage of training and gradually decreases to avoid error oscillations. It allows the neural network to find out the optimum solution quickly.

The neural network model performs predictions for the data analysis module and compares with the known data. By tuning the model, the user is able to explore, query and check the information that the analysis module provides. Once the data analysis module is set up and well tuned, the system can produce the reasonable analyzed results. This module uses the integration services between data analysis and reporting in SQL server 2005, providing predictive results in a flexible and scalable manner to the end users. Figure 7 shows an odour strength analysis interface using an artificial neural network approach. Figure 8 shows a comparison between the prediction results and the actual measured values of odour strength, where the predicted values match the actual ones very well. The actual measured odour strengths range from 0 to $60 \mathrm{TD}$ (dilution to threshold), and the mean square error of the predicted odour strengths against the measured ones is 1.46 . It demonstrates that the data analysis module produces accurate predictions. Figure 9 shows an example of the result report. A textual or graphic report is generated to show the patterns about the prediction. After the data analysis process is complete and an accurate result is created, the application generates userfriendly reports. The report will be displayed on the browser screen or be deployed to Microsoft Office. This reporting module allows the data analysis results to be easily delivered to users in a simple format. 


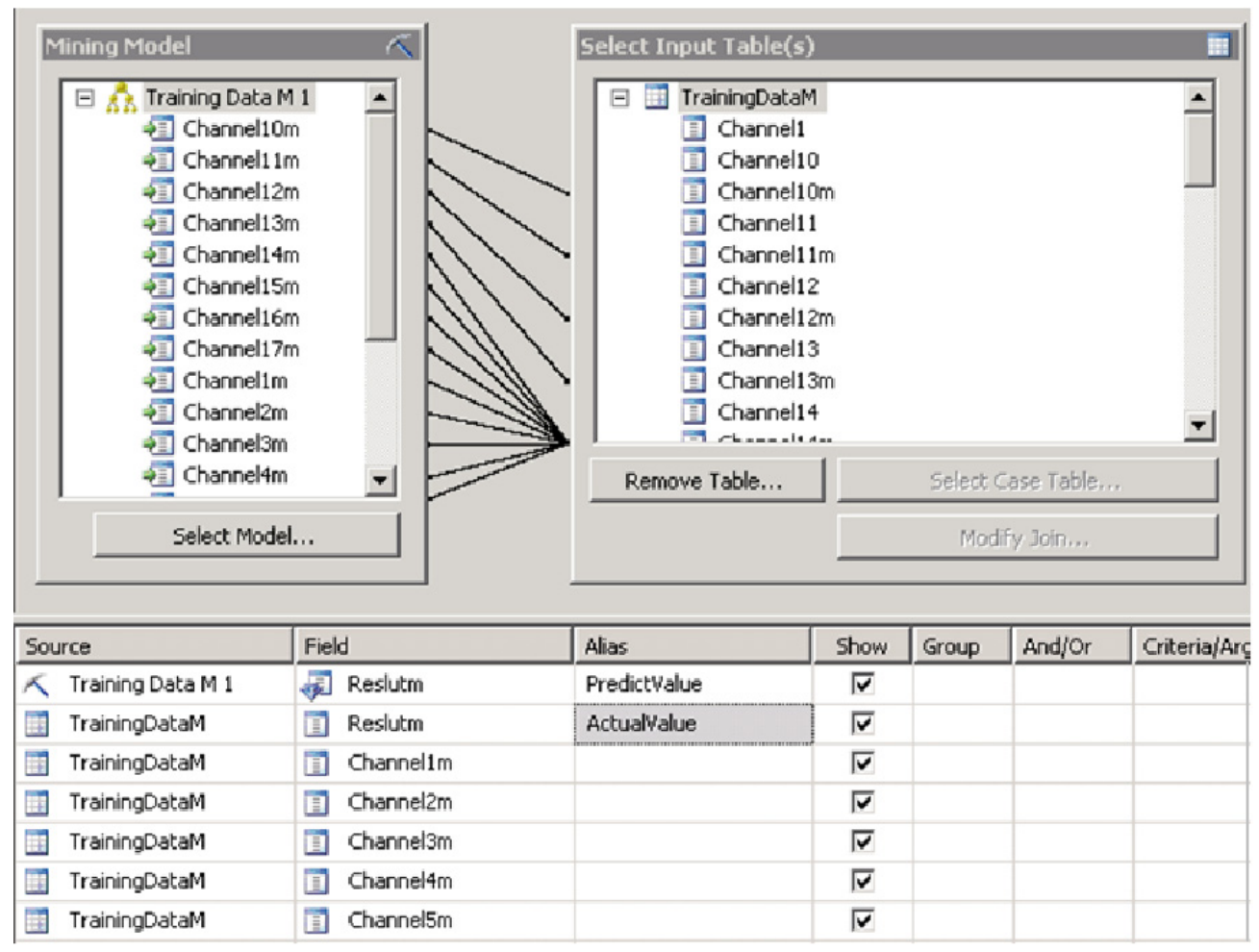

Figure 7. Odour strength analysis module.

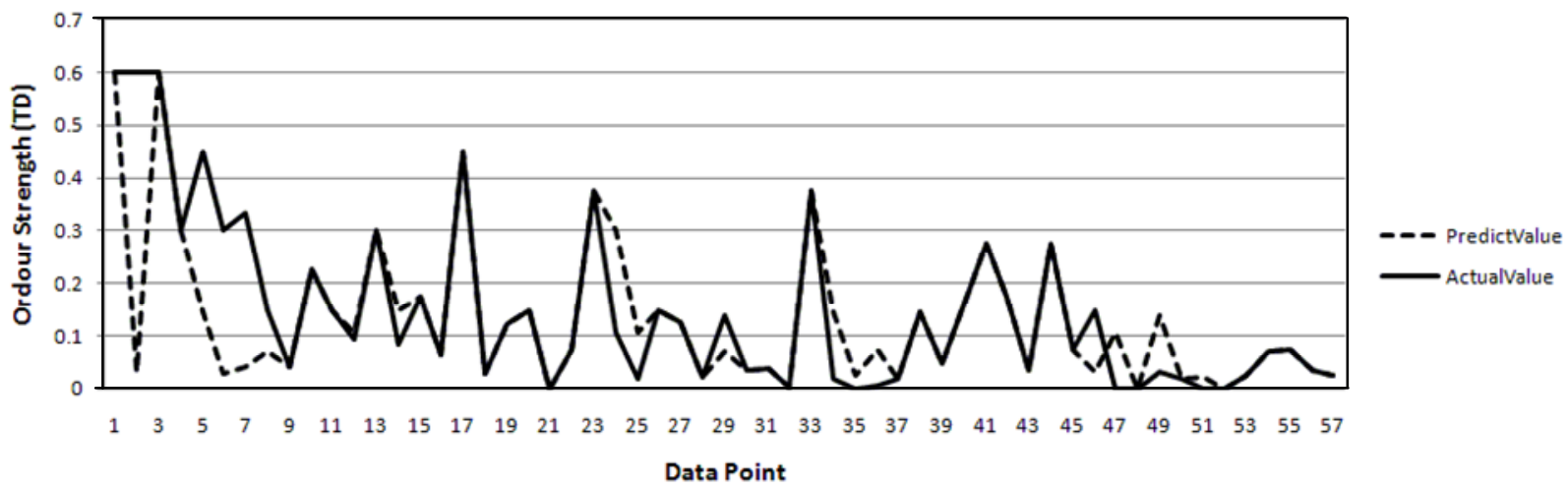

Figure 8. A comparison between the predicted value and actual value of odour strength.

\section{(2) Workflow Management}

The sensor data processing requires the workflow management to manage the analyzing processes, record submission, processing data, routing, etc. This central application server implements a comprehensive workflow, by using Microsoft Workflow Foundation (WFF) technology. This technology has support for complex workflow functionality, rich user interface, long running processes and advanced multiple level custom actions. The collaborative system has the seamless supports for WFF. The solution is integrated with the user interactive functionality and is built up on it. The workflow integration feature can be used to provide custom workflow for any custom business process/application. Users in research community can collaborate on data records and manage data processing tasks through particular web forms. Workflows help users to adhere to consistent project processes.

\section{(3) Personalization and Targeting}

Personalization and targeting component is used to target user specific information. This component primarily reads information from existing data sources, and retrieves changes to user data in the parent data sources (i.e. Active Directory), and then synchronizes with the system user profile database. The system user profile database is used for managing user profiles. All the publishing interactions with user profiles, audiences and alerts are based on the proposed personalization and targeting component. Personalization and content targeting component are exposed as a functional web service, which 


\begin{tabular}{|c|c|c|c|c|c|c|}
\hline$\mathbb { 4 } \triangleleft \longdiv { 1 }$ & of 16 & & $100 \%$ & 7 & Find I Next & Select a format \\
\hline \multicolumn{7}{|c|}{ Report July } \\
\hline Result & Channell & & Channel2 & Channel3 & Channel4 & Channel5 \\
\hline 2.17 & & & 1232 & 547 & 1308 & 358 \\
\hline 2.17 & & & 1232 & 547 & 1305 & 359 \\
\hline 60 & & & 1231 & 546 & 1305 & 361 \\
\hline 2 & 1 & & 1232 & 544 & 1305 & 358 \\
\hline 2.17 & 1 & & 1233 & 547 & 1305 & 360 \\
\hline 0 & & & 1232 & 546 & 1306 & 361 \\
\hline 12.47 & 1 & & 1232 & 548 & 1305 & 360 \\
\hline 2.17 & & & 1233 & 547 & 1305 & 358 \\
\hline 2.17 & & & 1232 & 548 & 1305 & 357 \\
\hline 2 & & & 1233 & 547 & 1305 & 358 \\
\hline 2.17 & & & 1231 & 546 & 1306 & 358 \\
\hline 60 & & & 1232 & 544 & 1306 & 359 \\
\hline 12.47 & & & 1229 & 548 & 1305 & 357 \\
\hline
\end{tabular}

Figure 9. Odour data analysis result report.

allows users to control the audience to whom the content is targeted. Collaborative web site administrators (not necessarily domain or local administrators) can easily create these audiences. These audiences are logical groups that satisfy some filtering rules (AD attribute values or custom attributes) in user profile database. It should be noted that personalization and targeting are not the same as security and restricting access to content. Results management interface is an example of a content-specific functionality that controls the targeting of result reporting to specified audience. The personalization and targeting feature provides functionality such as display a result item (i.e. reports, announcements and etc) to authorized users only. The different audience can be identified by looking up the user roles.

\section{(4) Security}

All the applications and application servers within the collaborative system architecturally support Windows integrated authentication. Users are authenticated against their credentials and access rights as defined in the Active Directory server. All the applications make use of these user credentials and access rights to restrict specific content from being viewed by unauthorized users. Generally, the roles of a user (i.e. the Active Directory group that they belong to) determine the access permissions that he has on the collaborative web site.

\section{(5) User Interface}

Users interact with the collaborative framework system directly through a series of user interfaces. These interfaces are within the web-based interface application. The interface application provides front-end pages to the data record libraries, analysis result reports, discussion forums, task management, workflow management and other components. The homepage is the first displayed page when users start the application. Users log into the system from this page to get the access to other functional pages such as data record page and result reporting page. The security model of the sub-pages is inherited from the homepage so that it only needs one time login when browsing through the web pages. Figure 10 shows the homepage of the system. Users can navigate through the web site easily and find the information and content they need.

All task status can be monitored on the task status page. The task status control shows the task status of submitted requests, and the service status control shows the status of pending service requests of the current user. Status bar shows the completed percentage of the whole process. This page provides a way to track and close monitoring process status and participants.

The public discussion forum page is a place for the users to discuss topics of their interest and to interact with each other. Livestock farmers can consult with agencies or governments to improve their odour control operations; agencies can post their comments and concerns, rural residents can deliver their complaints about odour issue. The interface of the discussion forum is shown in Figure 11. 


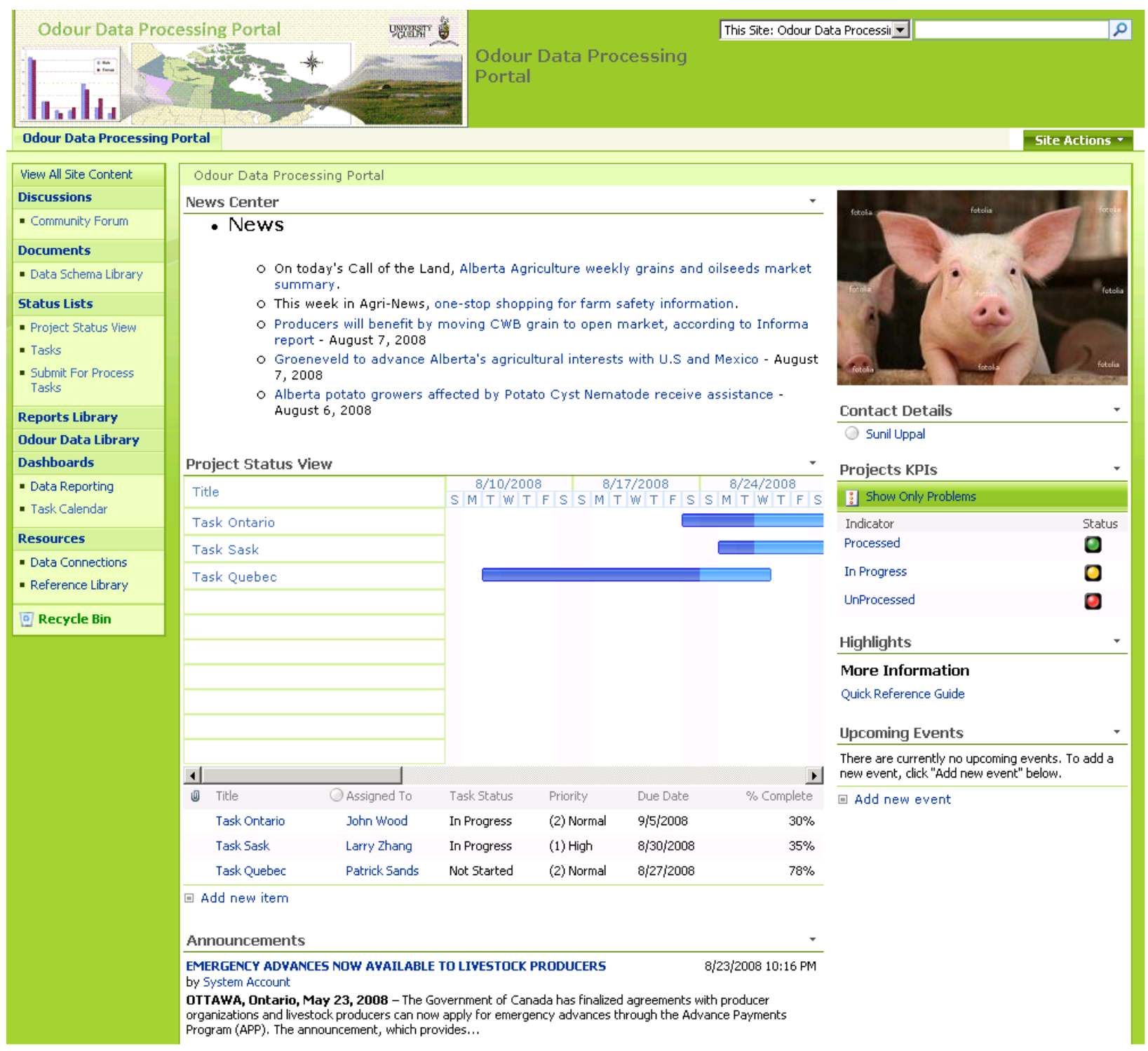

Figure 10. Homepage of the collaborative portal.

\section{Conclusions}

A collaborative portal framework is developed for acquisition, transmission, integration, management, processing and analysis of livestock odour data; and for providing a platform to enhance collaboration in livestock farm odour management. The user-friendly environment makes the application easy to use. The centralized data storage and analysis tools and models enable easy maintenance and improvement for the application. The authentication and authorization features provide enhanced security for the application. The web-based collaborative system is an efficient and convenient platform for collaboration among livestock producers, environment agencies, and governments. It not only brings a data integration solution to the research community but also increases the productivity of all the participants and brings significant added values to the data. The collaborative portal system eliminates the limitation on the livestock farm odour emission research by the Internet-accessible collaborative portal web site. The system delivers a collaborative interface to let users easily manage the process and share the data. The interface links researchers together based on the same interests about the experimental data, topics or projects. Researchers are no longer required to be in the same place working on the same project. The collaborative portal framework provides essential collaboration capabilities and dynamic, accurate, and robust environment information that promotes faster and more insightful decision making on livestock farm odour control problems.

Acknowledgments. This work is supported by Ontario Pork and National Science Engineering Research Council (NSERC). 


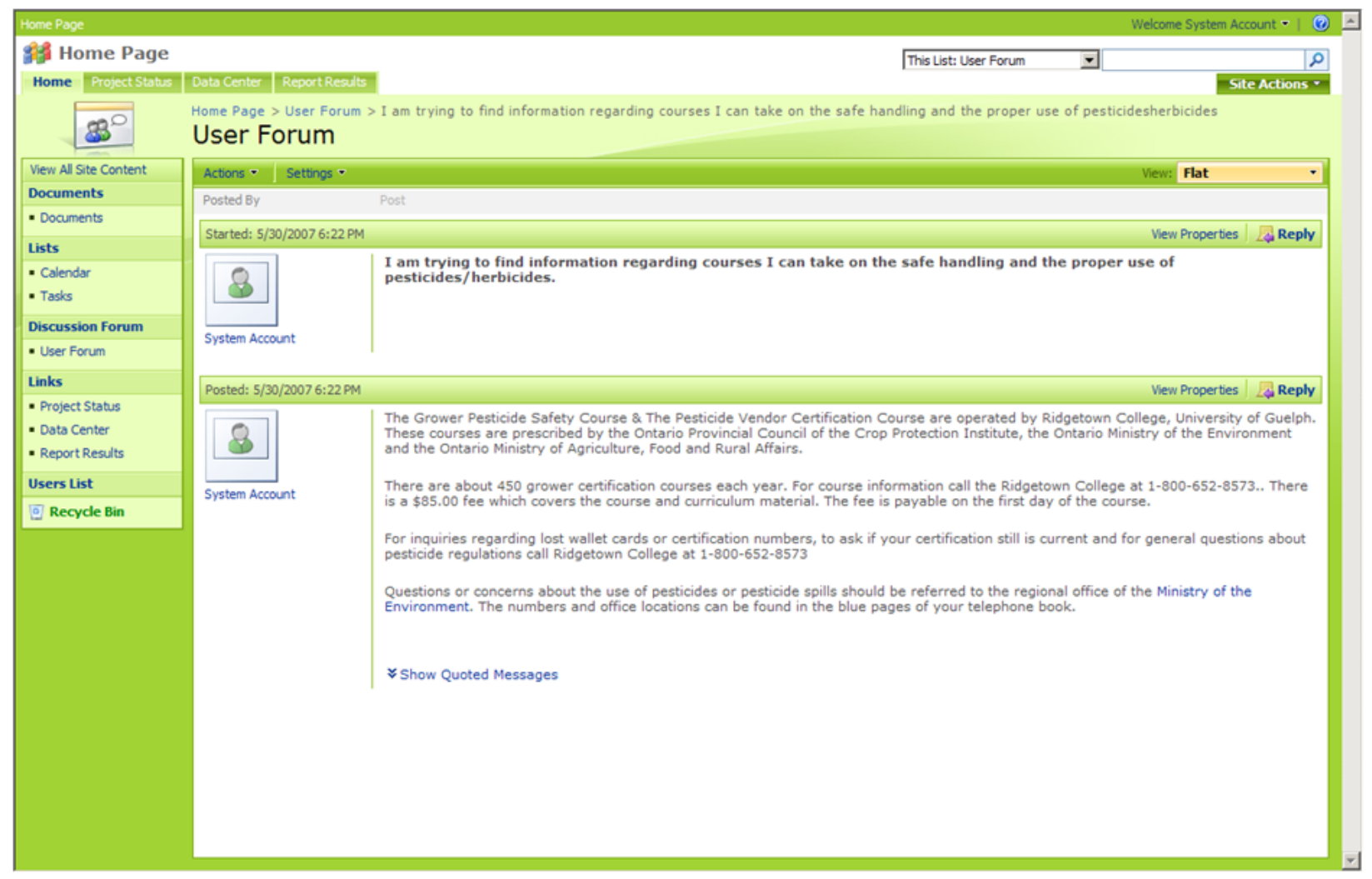

Figure 11. Public discussion forum.

\section{References}

Athanasiadia, I.N., and Mitkas, P.A. (2007). Knowledge discovery for operational decision support in air quality management, J. Env. Inform., 9(2), 100-107, doi:10.3808/jei.200700091.

Babar, M.A., and Gorton, I. (2004). Supporting architecture evaluation process with collaborative applications, Proceedings of INMIC 2004 8th International Multitopic Conference, pp. 440446.

Carswell, J.D., Gardiner, K., Bertolotto, M., Rizzini, A., and Mandrak, N. (2008). A web-based and mobile environmental management system, J. Env. Inform., 12(1), 9-20, doi:10.3808/jei.200800119.

Chang, K.E., Sung, Y.T., Wang, K.Y., and Dai, C.Y. (2003). Web-Soc: a socratic-dialectic-based collaborative tutoring system on the Workd Wide Web, IEEE Transactions on Education, 46(1), 69-78, doi:10.1109/TE.2002.808276.

Cheng, C.T., Chau, K.W., Gang, L., and Li, X.Y. (2005). Collaborative web application for flood control system of reservoirs, Web Technologies Research and Development-APWeb, 3399, 364374, doi:10.1007/b106936.

Counsell, J. (2004). "Under construction": the potential of a webbased application in managing and maintaining large area urban modeling. Cooperative Design, Visualization, and Engineering, 3190, 148-155.

Haykin, S. (1999). Neural Networks: a Comprehensive Foundation, Prentice Hall, NJ, USA.

Jackson, M.E. (2003). Portals: a strategic new direction to improve access to information resources, VINE, 33(1), 33-36, doi:10.1108 /03055720310698443

Janes, K.R., Yang, S.X., and Hacker, R.R. (2004). Single component modelling of pig farm odour with statistical methods and neural networks, Biosystems Eng., 88(3), 271-279, doi:10.1016/j.biosy stemseng.2004.04.004.

Janes, K.R., Yang, S.X., and Hacker, R.R. (2005). Pork farm odour modelling using multiple component multiple-factor analysis and neural networks, Applied Soft Computing, 6(1), 53-61, doi:10.1016 /j.asoc.2004.10.006.

Maglogiannis, I., Delakouridis, C., and Kazatzopoulos, L. (2006). Enabling collaborative medical diagnosis over the Internet via peer-to-peer distribution of electronic health records, J.Medicine Sys., 30, 107-116, doi:10.1007/s10916-005-7984-1.

Oxford University Press (1989). Oxford English Dictionary, 2nd Edition, Oxford University Press, UK.

Pan, L., Yang, S.X., Otten, L., and Hacker, R.R. (2006). Component and factor analysis of pork farm odour using structural learning with forgetting method, Biosystems Eng., 94(1), 85-93.

Pan, L., Yang, S.X., and DeBruyn, J. (2007). Factor analysis of downwind odours from livestock farms, Biosystems Eng., 96(3), 387397, doi:10.1016/j.biosystemseng.2006.10.017.

Pan, L., and Yang, S.X. (2007a). A new intelligent electronic nose system for measuring and analyzing livestock and poultry farm odours, Environ. Monitoring Assess., 135(1-3), 399-408, doi:10. 1007/s10661-007-9659-5.

Pan, L., and Yang, S.X. (2007b). Analyzing livestock farm odour using an adaptive neuro-fuzzy approach, Biosystems Eng., 97(3), 387-393, doi:10.1007/11816171.

Pan, L., and Yang, S.X. (2009). An electronic nose network system for online monitoring livestock farm odors, IEEE Transactions on Mechatronics, in press.

Taguchi, M., and Tokuda, T. (2005). Automatic generation of clientserver collaborative web applications from diagrams, Web Eng., 3597, 609-611, doi:10.1007/11531371 82.

Wang, J.W., Zhang, J.M., Wei, X.P., and Wang, J. (2004). Collabora- 
tive evaluation of engineering design concepts on the Web, Proceedings of the 8th International Conference on Computer Supported Cooperative Work in Design, pp. 224-229.

Yu, F.C., Chen, C.Y., Lin, S.C., Lin, Y.C, Wu, S.Y., and Cheung, K.W. (2007). A web-based decision support system for slopeland hazard warning, Environ. Monit. Assess., 127(1-3), 419-428.
Yu, H., Zhang, J., Wang, L., and Barksdale, J. (2003). A secure web application: 3D visualization and collaboration. Proceeding of Communications, Internet, and Information Technology, 408.

Zeng, W.H., Zhang, Y.J., Liu, J.L., Yang, Z.F. (2007). Web-Based Geographic Information System for UWRSR Evaluations, J. Env. Inform., 10(2), 75-81, doi:10.3808/jei.200700102. 